\title{
Email Reminder System
}

\author{
Vaibhav Maheshwari \\ Department of Computer Science \\ and Technology \\ Meerut Institute of Engineering and \\ Technology
}

\author{
Kashish Saini \\ Department of Computer Science \\ and Technology \\ Meerut Institute of Engineering and \\ Technology
}

\author{
Sarthak Gupta \\ Department of Computer Science \\ and Technology \\ Meerut Institute of Engineering and \\ Technology
}

\author{
Shivam Kumar \\ Department of Computer Science and Technology \\ Meerut Institute of Engineering and Technology
}

\author{
Swati Jain \\ Department of Computer Science and Technology \\ Meerut Institute of Engineering and Technology
}

\begin{abstract}
Email is a method of official communication in business giants, hospitals, lawyers, Small firms, banks, IT companies and much more. There are lots of emails in an inbox, few are important and more of them are unimportant, by which important mails get skipped many a time. So, our task is to provide a reminder to the users on their emails by which they can be reminded automatically at a specific date. Email Reminder provides flexibility to busy people who are not able to remember all our daily emails. So, this system also helps in the reminder of time-sensitive emails such as form filling mails, exam related mails, etc. And so, it becomes a perfect tool for business and personal follow-ups and also for the new generation who have not time to remember the things and living their lives in running mode.
\end{abstract}

\section{Keywords}

Email Reminder, SMTP, POP, Receive, Send, Web application, Database, Mail

\section{INTRODUCTION}

Today, within the time of the World Wide Web, emails needed to be updated and customized for his or her more importance and use in our daily lifestyle. One must be alerted for a crucial meeting, appointment, and other important emails. Communication through email has become common during this era, it's important to make a reminder application, by which one are often alerted through reminder.

This project aims to develop a reminder application that can store email reminders and will display an email at the time specified by the user. The user has to set a reminder for the mail in the inbox that he/she gets in the future automatically at the specified date and time as set by the user in the calendar. User friendly \& quick to use interface. It also saves the memory as the user deletes the content which is not for use.

The email reminder system is an application that provides a simple interface for reminders of email according to user choice, with the application user can easily manage their reminders like updating, deleting, or adding more reminders. The user has to set a reminder for their mails in an inbox that he/she gets in the future automatically at the specified date and time as set by the user in the calendar.

The email reminder system is the middleware system between the user and software that enables the user to set their reminders on emails and get them when times come, automatically. With the help of this project, users save their time in not searching the mail in their inbox.

\section{RELATED WORK}

A Medical Reminder System has an messaging watch which is programmed with a medicine regimen for the patient during which each watch is programmed with the patient's name, individual medicine names and specified medication times, also like alerts for appointments and medical events [1, 2].

In document reminder system, when action should be taken on a selected document, and also provides notation to the user indicating the acceptable course of action to be taken. It provides a calendaring feature where a Supervisor or manager can determine the workload of workers for an upcoming period of time [3]. A system and method for filtering communications between Correspondence Address: a sender and a recipient supported information stored in database. The system comprises a client program, an internet utility program and an internet interface for entering to the database [4].

The Set of notification preferences may indicate a desired notification medium through which the notification message is to be sent, a notification addressee to which the notification message is to be sent, and/or a notification timing preference indicating a date during which the notification is to be sent (or received) [5].

An electronic reminder System comprising a computer connectable to the web for receiving email messages through POP and/or SMTP [6]. When we have studied these research papers we expect we will also set reminder alerts for important mails. 


\section{METHADOLOGY}

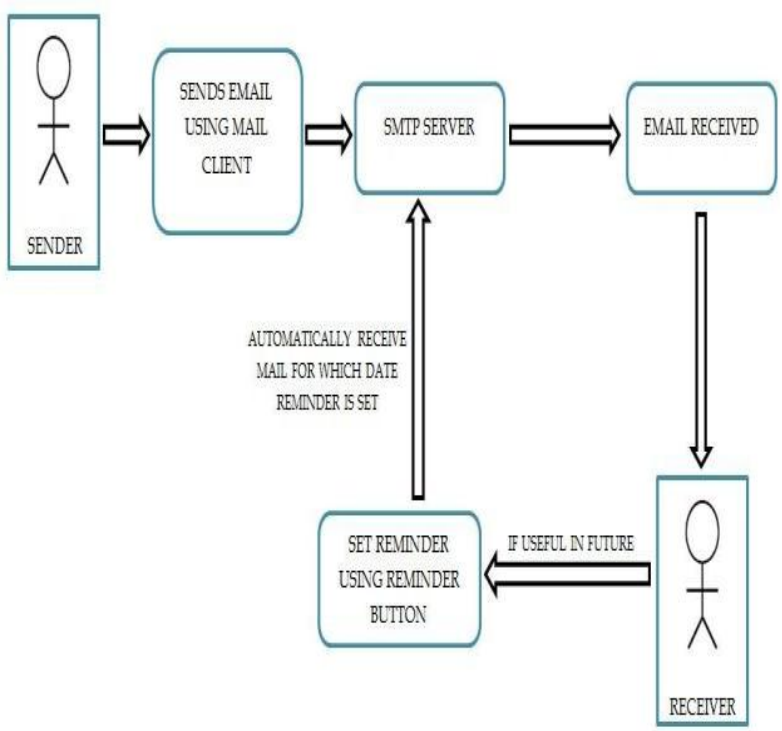

Fig 1: Work Flow Diagram

- This Project provides an interface which consists of reminder button.

- User selects a particular mail in the inbox and clicks the reminder button.

- A calendar opens, from the given calendar user can select a date in the calendar on which he wants a reminder.

- On the same date the selected mail again reminder pops up in the mailbox.

\section{MODULES}

1. Mark Important: In this module, we have to mark the important mails, which can be seen in important section. This is the following pseudo code:

1.1 Declare a String un $=($ String $)$ session.getAttribute $(" \operatorname{logn} ")$

1.2 Declare another String impmsg = request.getParameter("imp")

1.3 Create Connection con $=$ dao.createconnection () .

1.4 PreparedStatement ps 1 = con.prepareStatement("update mailsender_inbox set important='true' where receiver=? and datetime=?")

1.5 ps1.setString $(1$, un $)$ sets the receiver name to database.

$1.6 \mathrm{ps} 1$.setString(2,impmsg) sets the date and time to database.

$1.7 \mathrm{ps} 1$. executeUpdate() is used to apply the changes in database.

close connection.

1.8 response.sendRedirect("inbox.jsp") is used to show the output on inbox.jsp.

2. Set Reminder: This module is used to set the remider using calendar pop up, This is the following pseudo code:

2.1 function cat(form)

var settime $=$ document.signupform. settime.value

\author{
var $\mathrm{msg}=$ document.signupform.msg.value \\ if (settime.length $==0$ ) \\ alert("Error! Please Select Reminder Date") \\ document.signupform.settime.focus() \\ return false \\ else if(msg.length==0) \\ alert("Error! Reminder Message can't leave blank") \\ document.signupform.msg.focus() \\ return false \\ return true
}

3. Save Reminder: This module is used to save reminder and the reminder comes to specifies date. This is the following pseudo code:

3.1 Declare a String un $=($ String $)$ session.getAttribute $($ "logn")

3.2 Declare a String settime = request.getParameter("settime")

3.3 Declare a String msgid = request.getParameter("msgid")

3.4 Declare a String msg = request.getParameter("msg")

3.5 Create Connection con $=$ dao.createconnection();

3.6 PreparedStatement ps $=$ con.prepareStatement("update mailsender_inbox

3.7 ps.setString $(1$, settime $)$ is used to set time in database.

3.8 ps.setString $(2, \mathrm{msg})$ is used to set message in database.

$3.9 \mathrm{ps}$.setString $(3$,un $)$ is used to set receiver name in database

3.10 ps.setString(4,msgid) is used to set message id.

$3.11 \mathrm{ps}$. executeUpdate() is used to update the values in database.

close connection.

3.12 response.sendRedirect("important.jsp")

\section{RESULT DISCUSSION}

The Email Reminder System was successfully implemented and deployed to the environment and it is planned to be released in the incoming Program Increment. All components have passed their Unit Tests and their respective Acceptance Tests, after executing these tests we have seen no issue within the tests performed. All the proposed functions worked fine and therefore the notifications (SMS) has been sent and checked at different period of your time. 


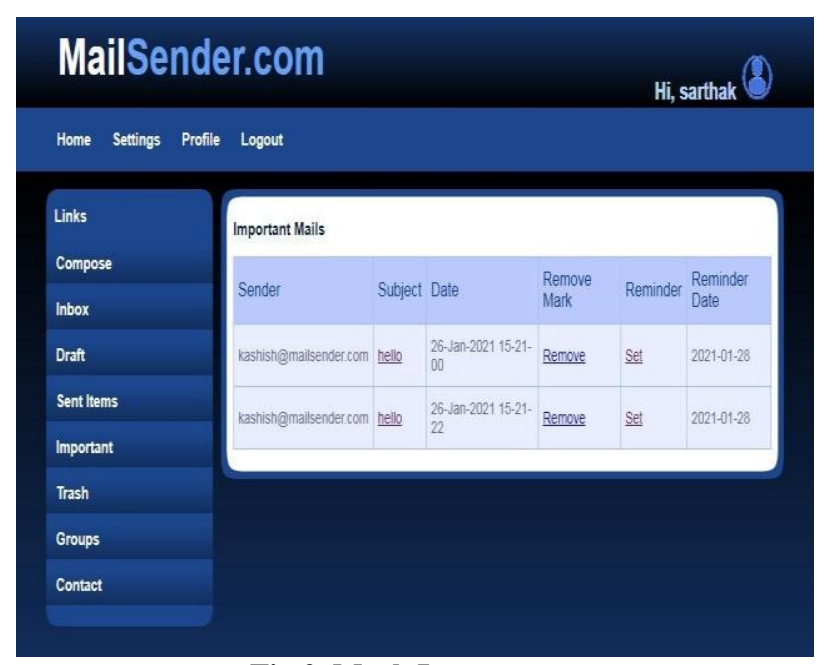

Fig 2. Mark Important

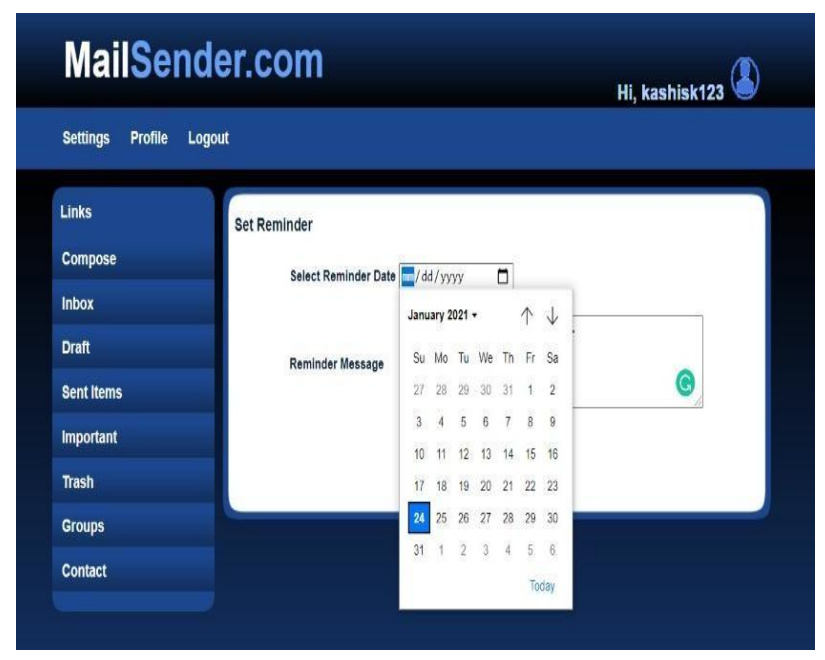

Fig 3. Set Reminder

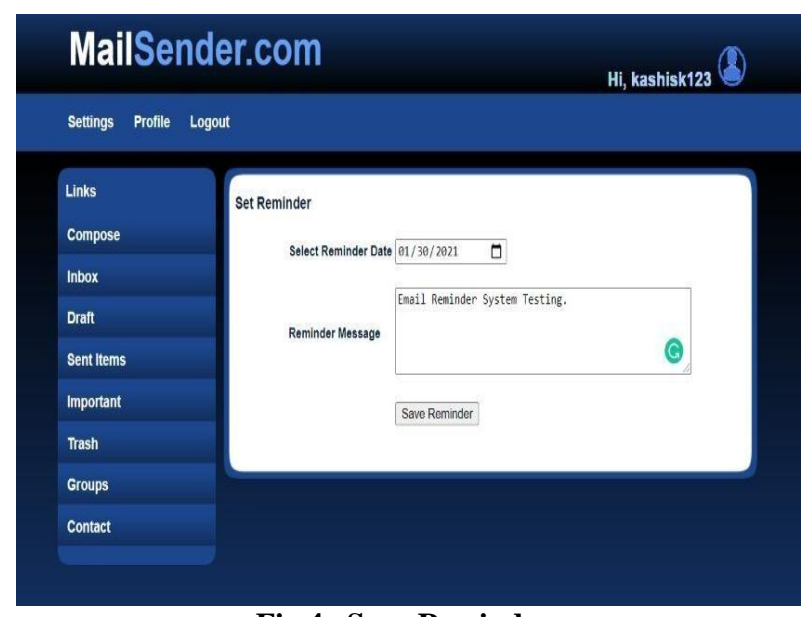

Fig 4. Save Reminder

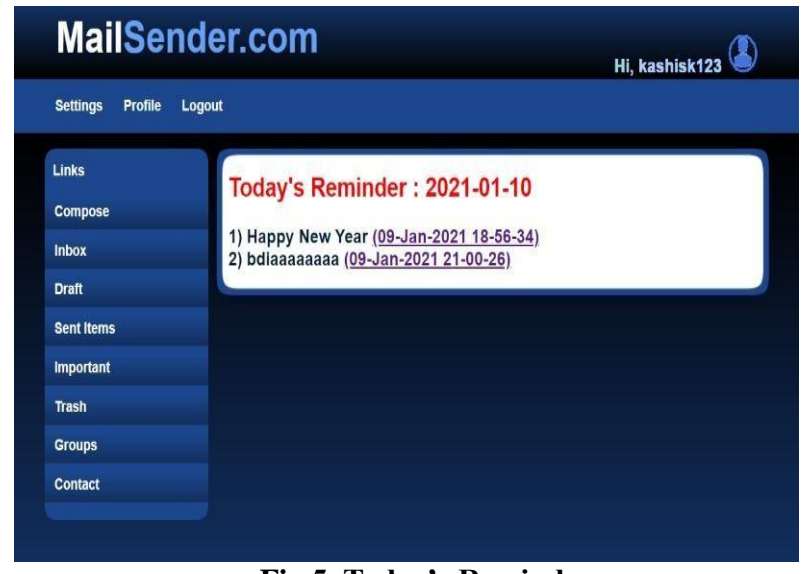

Fig 5. Today's Reminder

\section{CONCLUSION}

Nowadays, mails play a most important role in our life. We receive lots of emails in a day but we are unable to remember all important mails regarding meetings, exams, assessments, academic lectures, assignments and interview mails, etc. So, we face problems regarding remembering the emails, and note them down somewhere and make a note is not a good option because then we have to track these complex notes. It also helps in reducing time required to manage calendar, also groups can be created which reduces time for sending mails multiple times.

So, we conclude that mail reminder system plays a more important role in our daily life by which user can place a reminder on important mails and system gets remind to the user when the times come automatically.

\section{REFERENCES}

[1] David Zarchan, Concord, Mass., "Medical reminder system and messaging watch", United States Patent, 6,075,755, Jun. 13, 2000

[2] Kent P. Steinnagel, Larchmont, N.Y., "Reminder system for taking medication", United States Patent, 4,974,729, Dec. 4, 1990

[3] Bruce L. Karlson, Mission, KS (US), "Document reminder system", United States Patent, US 8,365,080 B2, Jan. 29, 2013

[4] Alberto Mujica, Miami, FL (US), "E-MAIL SYSTEM", United States Patent, US 2006/0253597 A1, Nov. 9, 2006

[5] Kevin Hsiaohsu Tu, Milpitas, CA (US), "Event notification system", United States Patent, US 6,617,969 B2, Sep. 9, 2003

[6] Harold F. Feinleib, "Electronic reminder system with universal email input", United States Patent, US 6,272,532 B1, Aug. 7, 2001

[7] Java development tools in eclipse, JDT. (Online) Available: http://www.eclipse.org/jdt/ [Accessed: 26 December 2020]

[8] Reynaldo Burgos Torres, "Event Notification System".

[9] Wikipedia, Email, Wikipedia the free encyclopedia.https://en.wikipedia.org/wiki/Email. 